\title{
Avaliação técnico-econômica de suínos machos imuno e cirurgicamente castrados
}

\author{
Technical-economic evaluation of imuno and surgically castrated male pigs
}

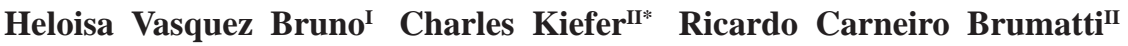 \\ Alexandre Pereira dos Santos ${ }^{\mathrm{II}}$ Gabriel Cipriano Rocha ${ }^{\mathrm{III}}$ Gabriela Puhl Rodrigues ${ }^{\mathrm{II}}$
}

RESUMO

Realizou-se este estudo com o objetivo de avaliar o desempenho técnico-econômico e determinar a lucratividade, por meio da análise de sensibilidade econômica, de suínos machos imuno (IM) e cirurgicamente castrados (MC). Foram realizados dois experimentos, em delineamento inteiramente casualizado, composto por dois tratamentos (IM e MC), com oito repetições de 10 animais cada, totalizando 160 suínos por experimento. Verificou-se que suínos IM apresentam menor consumo de ração e melhor conversão alimentar nas fases de crescimento em relação aos MC. Na fase de terminação, suínos IM apresentam melhor ganho de peso, conversão alimentar, rendimento de carcaça e porcentagem de carne em relação aos MC. A utilização de IM aumenta a lucratividade na produção de suínos. A análise de lucratividade entre suínos IM e MC deve considerar todas as fases de criação, uma vez que o desempenho diferencial dos suínos não castrados na fase de crescimento influencia economicamente os resultados da produção.

Palavras-chave: análise de sensibilidade, carcaça, imunocastração, lucro.

\section{ABSTRACT}

This study was conducted to evaluate the technical and economic performance and determine profitability through the economic sensitivity analysis of immune (IM) and male pigs surgically castrated (SC). Two experiments were conducted in a completely randomized design, consisting of two treatments (IM and SC) with eight replicates of 10 animals each, totaling 160 pigs per experiment. It was found that IM pigs have lower feed intake and feed conversion during the growth phases in relation to SC. In the finishing phases, IM have better weight gain, feed conversion, carcass yield and lean meat percentage in relation to SC. The use of IM increases the profitability in pig production. The analysis of profitability differential between IM and SC should consider all stages of creation, since the differential performance of not castrated pigs during growth influences economic results of production.

Key words: sensitivity analysis, carcass, immunocastration, profitability.

\section{INTRODUÇÃO}

Suínos machos não castrados apresentam melhor conversão alimentar e carcaças com maior percentual de carne do que os MC (BONNEAU, 1998), porém a castração é necessária, devido às altas concentrações de androsterona e escatol acumuladas na gordura subcutânea e intramuscular de machos não castrados (PATTERSON, 1968). A imunocastração induz a formação de anticorpos contra o hormônio liberador de gonadotropina $(\mathrm{GnRH})$, que se ligam ao GnRH endógeno, bloqueando o estímulo à secreção do hormônio folículo estimulante (FSH) e do hormônio luteinizante (LH), causando redução da secreção de esteroides testiculares, incluindo androsterona (ZAMARATSKAIA et al., 2008). Esse fato possibilita a produção de carcaças isentas de odores sexuais (JAROS et al., 2005).

Suínos castrados imunologicamente apresentam melhor eficiência alimentar e carcaças com maior percentual de carne que suínos MC (PAULY et al., 2009). Também se observa a redução da frequência de montas e do comportamento agressivo dos IM, quando comparados aos não castrados (RYDHMER et al., 2010). Nesse sentido, atendendo

\footnotetext{
IPrograma de Pós-graduação em Ciência Animal, Universidade Federal de Mato Grosso do Sul (UFMS), Campo Grande, MS, Brasil.

IIUFMS, 97070-900, Campo Grande, MS, Brasil. E-mail: charles.kiefer@ufms.br. *Autor para correspondência.

IIIPrograma de Pós-graduação em Zootecnia, Universidade Federal de Viçosa (UFV), Viçosa, MG, Brasil. Recebido 21.12.12 Aprovado 29.05.13 Devolvido pelo autor 08.08.13 CR-2012-1343.R1
} 
ao crescente interesse mundial em torno do bem-estar animal, a imunocastração pode reduzir o sofrimento e estresse causados pela castração cirúrgica.

Em vista dos benefícios que a imunocastração pode oferecer à suinocultura, realizou-se este estudo com o objetivo de avaliar o desempenho técnico-econômico e determinar a lucratividade, por meio da análise de sensibilidade econômica, de suínos IM e MC.

\section{MATERIAL E MÉTODOS}

Foram realizados dois experimentos com suínos IM e MC, da mesma linhagem. No experimento I, foram utilizados 160 suínos (80 IM e $80 \mathrm{MC}$ ), avaliados dos $95,8 \pm 5,5 \mathrm{~kg}$ aos $122,9 \pm 5,1 \mathrm{~kg}$, com período experimental de 30 dias. No experimento II, foram utilizados 160 suínos ( 80 IM e 80 MC), avaliados dos $27,5 \pm 1,0 \mathrm{~kg}$ aos $114,5 \pm 6,3 \mathrm{~kg}$, com período experimental de 98 dias. Em cada experimento, os animais foram distribuídos em delineamento experimental inteiramente casualizado, com dois tratamentos (IM e MC), com dez repetições e oito animais cada. As dietas experimentais foram elaboradas de acordo com as recomendações de ROSTAGNO et al. (2005) e fornecidas à vontade aos animais.

Os suínos $\mathrm{MC}$ foram submetidos à orquiectomia até o sétimo dia de vida. Para a obtenção dos IM, os machos receberam duas doses de vacina anti-GnRH, via subcutânea, 60 e 30 dias pré-abate. As variáveis avaliadas foram o desempenho (peso final, consumo de ração, ganho de peso e conversão alimentar) e as características de carcaça (peso e rendimento de carcaça e percentual de carne). Para a avaliação econômica, as variáveis utilizadas foram o custo das dietas $\left(\mathrm{R} \$ \mathrm{~kg}^{-1}\right)$, custo da imunocastração, preço do suíno vivo $\left(\mathrm{R} \$ \mathrm{~kg}^{-1}\right)$, receita de bonificação $(\mathrm{R} \$)$ e lucro bruto (R\$). As equações de custo da dieta foram: Custo da dieta $(\mathrm{R} \$$ Dieta $)=($ Consumo $) *(\mathrm{R} \$$ $\mathrm{kg}^{-1}$ da dieta)*(Período).

O preço comercial estabelecido para a dose da vacina foi de $\mathrm{R} \$ 5,00$. Considerando que são necessárias duas doses por animal, o custo da imunocastração foi de R $\$ 10,00$ por cabeça. O custo total $(\mathrm{CT})$ foi calculado pela fórmula: $\mathrm{CT}=(\mathrm{R} \$$ Dieta $)+($ Custo da Imunocastração). Para a obtenção da receita pelo sistema de bonificação $(\mathrm{BF})$, foi utilizada a equação proposta por GUIDONI (2000): Receita $\mathrm{BF}=\mathrm{R} \$ / \mathrm{PV}^{-1 *}[(\mathrm{PC}$ arc $/ \%$ Rend $) *(23,6+0,2$ $86 * \mathrm{PCarc}+\%$ CarnM $)$, onde: $\mathrm{R} \$ \mathrm{PV}^{-1}=$ preço pago por quilograma de suíno; $\mathrm{PCarc}=$ peso de carcaça; $\%$ Rend $=$ percentual de rendimento de carcaça e $\%$ CarnM= percentual de carne da carcaça.
A diferença entre receitas e custos resultou no lucro bruto de cada sistema, considerado o cenário padrão do mercado. Não foram computados os demais custos de produção por serem considerados similares para a produção de suínos IM e MC. A diferença entre suínos IM e MC foi obtida por intermédio da fórmula: Diferença $(\mathrm{IM}-\mathrm{MC})=$ Lucro bruto IM - Lucro bruto MC. A diferença percentual foi obtida através da fórmula: Diferença \% (IM-MC) = Diferença (IM$\mathrm{MC}) /$ Lucro bruto MC.

Após a determinação dos resultados das equações, realizou-se a análise de sensibilidade, pressupondo a construção de três cenários: mais provável (M); otimista $(\mathrm{O})$; pessimista $(\mathrm{P})$ e denominada análise MOP (GITMAN, 2000). Esses cenários foram idealizados de acordo com a oscilação do custo da dieta, receita obtida com a bonificação de carcaça e custo da vacina. A variação utilizada no custo da dieta foi de $10 \%$, valor estabelecido de acordo com a média variação de preços dos principais ingredientes das dietas (milho e farelo de soja) durante o ano de 2011, obtida do indicador de preços CEPEA (2011).

A variação na receita de bonificação foi obtida através da média dos indicadores de preços do suíno $\left(2,40 \mathrm{R} \$ \mathrm{~kg}^{-1}\right)$, pago ao produtor no decorrer de 2011, em SP, MG, SC e RS, sendo observada uma variação média de 19\% (CEPEA, 2011). Para composição da variação de custos no valor da vacina, optou-se por uma oscilação de $10 \%$ no preço.

O cenário mais provável reflete o cenário padrão da produção em 2011. Para o cenário pessimista, foi utilizado aumento no custo de alimentação de $10 \%$, queda na receita de bonificação de $19 \%$ e aumento do custo da imunocastração de $10 \%$, em relação ao cenário mais provável. No cenário otimista, aplicou-se um decréscimo de $10 \%$ no custo de alimentação e aumento de $19 \%$ na receita de bonificação e decréscimo no custo da imunocastração de $10 \%$ (Tabela 1$)$.

Os dados zootécnicos foram submetidos à análise de variância, utilizando-se o peso inicial como co-variável. As eventuais diferenças entre as médias foram avaliadas pelo teste $\mathrm{F}$, por intermédio do procedimento GLM do programa estatístico SAS versão 9.1. Adotou-se o nível de $5 \%$ de probabilidade.

\section{RESULTADOS E DISCUSSÃO}

No experimento I, os suínos IM apresentaram maior $(\mathrm{P} \leq 0,05)$ ganho de peso, melhor conversão alimentar e maiores percentuais de carne e rendimento de carcaça em relação aos suínos $\mathrm{MC}$ 
Tabela 1 - Variação de custos de acordo com os cenários por animal.

\begin{tabular}{llcr}
\hline Experimentos & Receitas e Custos & Mais Provável & Otimista \\
\hline Experimento I & Dieta terminação, $\mathrm{R} \$ \mathrm{~kg}^{-1}$ & 0,57 & 0,51 \\
Experimento II & Dieta crescimento I, $\mathrm{R} \$ \mathrm{~kg}^{-1}$ & 0,64 & 0,58 \\
Experimento II & Dieta crescimento II, $\mathrm{R} \$ \mathrm{~kg}^{-1}$ & 0,63 & 0,56 \\
Experimento II & Dieta terminação, $\mathrm{R} \$ \mathrm{~kg}^{-1}$ & 0,61 & 0,70 \\
Experimentos I e II & Imunocastração, $\mathrm{R} \$$ dos $^{-1} \mathrm{e}$ & 5,00 & 0,69 \\
Experimentos I e II & Preço suíno vivo, $\mathrm{R} \$ \mathrm{~kg}^{-1}$ & 2,40 & 0,67 \\
\hline
\end{tabular}

(Tabela 2). O consumo de ração diário, peso final, peso e comprimento de carcaça, a espessura de toucinho e a profundidade de músculo não foram influenciados $(\mathrm{P}>0,05)$ pelas categorias.

No experimento II, fase crescimento I, os suínos IM apresentaram melhor $(\mathrm{P}<0,05)$ conversão alimentar em relação aos $\mathrm{MC}$, enquanto as demais variáveis avaliadas não foram influenciadas $(\mathrm{P}>0,05)$ pelas categorias (Tabela 3 ). Na fase de crescimento II, os suínos $\mathrm{MC}$ apresentaram menor $(\mathrm{P} \leq 0,05)$ consumo de ração diário em relação aos IM, porém as demais variáveis não foram influenciadas $(\mathrm{P}>0,05)$. $\mathrm{Na}$ fase de terminação, os suínos IM apresentaram maiores $(\mathrm{P} \leq 0,05)$ peso final e ganho de peso diário e menor $(P \leq 0,05)$ conversão alimentar, bem como maiores $(\mathrm{P} \leq 0,05)$ peso de carcaça e rendimento de carcaça quente, comprimento de carcaça, espessura de toucinho e percentagem de carne, em relação aos castrados cirurgicamente. Por sua vez, o consumo de ração diário e a profundidade de músculo não foram influenciados $(\mathrm{P}>0,05)$ pelas categorias.

A superioridade dos suínos IM em relação aos $\mathrm{MC}$, observada para a conversão alimentar, no presente estudo, pode ser explicada considerando que os suínos, previamente à imunocastração, mantêm o padrão metabólico dos machos não castrados, uma vez que o efeito inibidor da secreção de esteroides testiculares inicia somente após a aplicação da segunda dose da vacina (ZAMARATSKAIA et al., 2008). O resultado obtido neste estudo está de acordo com a literatura (XUE et al., 1997), em que se constata superioridade absoluta da eficiência alimentar dos machos não castrados em relação aos castrados. De modo similar, PAULY et al. (2009) observaram melhor conversão alimentar dos IM em relação aos MC.

Diversos pesquisadores como JAROS et al. (2005) e PAULY et al. (2009) relataram o aumento do consumo de ração diário na fase de terminação dos animais imunocastrados. Porém, no presente estudo, não foram constatadas diferenças $(\mathrm{P}>0,05)$ entre os grupos testados para esse parâmetro, na fase de terminação.

Os melhores resultados observados para o rendimento de carcaça e percentagem de carne dos suínos IM, nos experimentos I e II, também foram

Tabela 2 - Desempenho de suínos IM e MC na fase de terminação (Experimento I).

\begin{tabular}{|c|c|c|c|c|}
\hline \multirow{2}{*}{ Variáveis } & \multirow[b]{2}{*}{ IM } & \multirow[b]{2}{*}{$\mathrm{MC}$} & \multirow{2}{*}{ Valor P } & \multirow{2}{*}{$\mathrm{CV}(\%)$} \\
\hline & & & & \\
\hline Peso inicial, $\mathrm{kg}$ & 94,99 & 96,78 & - & - \\
\hline Peso final, $\mathrm{kg}$ & 124,22 & 121,38 & 0,148 & 3,07 \\
\hline Consumo de ração diário, $\mathrm{kg}$ & 3,43 & 3,28 & 0,391 & 10,67 \\
\hline Ganho de peso diário, $\mathrm{kg}^{*}$ & 0,97 & 0,81 & 0,026 & 14,40 \\
\hline Conversão alimentar* & 3,60 & 4,27 & 0,050 & 16,05 \\
\hline Peso de carcaça quente, $\mathrm{kg}$ & 105,82 & 103,76 & 0,194 & 7,44 \\
\hline Rendimento de carcaça, $\% *$ & 75,93 & 78,19 & 0,002 & 1,39 \\
\hline Comprimento de carcaça, $\mathrm{cm}$ & 101,50 & 100,75 & 0,649 & 2,17 \\
\hline Espessura de toucinho, $\mathrm{mm}$ & 11,17 & 14,50 & 0,114 & 19,21 \\
\hline Profundidade de músculo, $\mathrm{cm}$ & 64,50 & 61,92 & 0,275 & 4,72 \\
\hline Carne, $\% *$ & 58,93 & 57,47 & 0,040 & 2,29 \\
\hline
\end{tabular}

IM (machos imunocastrados), MC (machos castrados cirurgicamente).

*Efeito significativo $(\mathrm{P}<0,05)$. 
Tabela 3 - Desempenho de suínos IM e MC nas fases de crescimento I e II e terminação (experimento II).

\begin{tabular}{|c|c|c|c|c|}
\hline \multirow{3}{*}{ Variáveis } & & & \multirow{3}{*}{ Valor P } & \multirow{3}{*}{$\mathrm{CV}(\%)$} \\
\hline & & & & \\
\hline & IM & $\mathrm{MC}$ & & \\
\hline Peso inicial, $\mathrm{kg}$ & 27,86 & 27,19 & - & - \\
\hline Peso final, $\mathrm{kg}$ & 58,87 & 56,93 & 0,187 & 4,80 \\
\hline Consumo de ração diário, $\mathrm{kg}$ & 1,65 & 1,78 & 0,196 & 11,32 \\
\hline Ganho de peso diário, $\mathrm{kg}$ & 0,78 & 0,75 & 0,461 & 11,62 \\
\hline \multirow[t]{4}{*}{ Conversão alimentar* } & 2,12 & 2,37 & 0,050 & 11,20 \\
\hline & \multicolumn{3}{|c|}{ 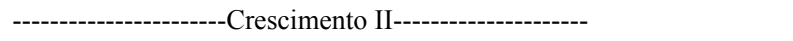 } & \\
\hline & & & Valor P & $\mathrm{CV}(\%)$ \\
\hline & $\mathrm{IM}$ & $\mathrm{MC}$ & & \\
\hline Peso final, $\mathrm{kg}$ & 81,55 & 79,89 & 0,198 & 3,76 \\
\hline Consumo de ração diário, $\mathrm{kg} *$ & 2,02 & 2,20 & 0,031 & 9,55 \\
\hline Ganho de peso diário, $\mathrm{kg}$ & 0,80 & 0,82 & 0,667 & 13,51 \\
\hline \multirow[t]{2}{*}{ Conversão alimentar } & 2,54 & 2,72 & 0,144 & 11,22 \\
\hline & \multicolumn{2}{|c|}{------------------------Terminação----------------------- } & Valor P & CV $(\%)$ \\
\hline Peso final, $\mathrm{kg}^{*}$ & 118,91 & 110,06 & 0,002 & 3,94 \\
\hline Consumo de ração diário, $\mathrm{kg}$ & 3,34 & 3,23 & 0,405 & 7,50 \\
\hline Ganho de peso diário, $\mathrm{kg}^{*}$ & 1,03 & 0,82 & 0,017 & 16,13 \\
\hline Conversão alimentar* & 3,26 & 4,18 & 0,025 & 19,48 \\
\hline Peso de carcaça quente, $\mathrm{kg} *$ & 93,55 & 88,68 & 0,015 & 3,83 \\
\hline Rendimento de carcaça, $\% *$ & 82,94 & 84,23 & 0,026 & 1,28 \\
\hline Comprimento de carcaça, $\mathrm{cm}^{*}$ & 100,25 & 96,80 & 0,006 & 2,14 \\
\hline Espessura de toucinho, $\mathrm{mm}^{*}$ & 8,75 & 11,20 & 0,023 & 19,19 \\
\hline Profundidade de músculo, cm & 67,43 & 65,04 & 0,375 & 7,89 \\
\hline Carne, $\% *$ & 61,67 & 60,01 & 0,014 & 1,91 \\
\hline
\end{tabular}

IM (machos imunocastrados), MC (machos castrados cirurgicamente).

* Efeito significativo $(\mathrm{P}<0,05)$.

relatados por outros pesquisadores (XUE et al., 1997; PAULY et al., 2009). Na prática, a maioria dos leitões machos é submetida ao procedimento cirúrgico para remoção dos testículos na primeira semana de vida. Esse procedimento resulta na ausência das funções anabólicas geradas pelos hormônios testiculares nas fases de recria e terminação (CAMPBELL et al., 1985). A ausência dos hormônios testiculares diminui o potencial de crescimento e aumenta a deposição de gordura na carcaça nos suínos MC (METZ \& CLAUS, 2003). Por sua vez, os suínos IM mantêm esse potencial anabólico durante a fase de recria e ainda por alguns dias após a aplicação da segunda dose da vacina, quando já estão na fase de terminação (CLAUS et al., 2007). Essas diferenças no padrão metabólico justificam a superioridade dos suínos IM, quando comparados aos MC, no presente estudo.

$\mathrm{Na}$ análise de sensibilidade para o cenário mais provável (Tabela 4), no experimento I, os animais IM apresentaram maior lucro bruto, resultando numa diferença monetária de R\$1,07 (0,04\%) em relação ao grupo MC. No experimento II, a lucratividade dos animais IM em relação aos MC foi superior àquela observada no experimento I, em que o lucro bruto dos suínos IM apresentou superioridade de $\mathrm{R}$ \$22,39 (16,98\%) em relação ao dos MC. Dessa maneira, ficou evidenciado o efeito do ganho acumulado durante as três fases da produção, uma vez que o desempenho zootécnico dos animais IM foi superior ao dos suínos MC desde a fase de crescimento I, resultando, consequentemente, em menor custo de alimentação.

$\mathrm{Na}$ análise de sensibilidade para o cenário otimista, os resultados seguiram o mesmo padrão do cenário mais provável. No experimento I, ocorreu uma diferença maior no lucro bruto para os animais IM, resultando numa diferença monetária de R $\$ 4,92$ $(1,47 \%)$ em relação ao grupo MC. No experimento II, a lucratividade dos animais IM em relação aos MC foi superior àquela observada no experimento I, em que o lucro bruto dos suínos IM apresentou superioridade de R\$ 28,25 (14,23\%) em relação aos MC. A maior diferença monetária no cenário otimista em relação ao cenário mais provável provavelmente está relacionada ao potencial diferencial de eficiência alimentar dos 
Tabela 4 - Análise de sensibilidade para os cenários mais provável, otimista e pessimista.

\begin{tabular}{|c|c|c|c|c|}
\hline \multirow{2}{*}{ Receita e custos } & \multirow[b]{2}{*}{ IM } & \multirow[b]{2}{*}{$\mathrm{MC}$} & \multirow[b]{2}{*}{ IM } & \multirow[b]{2}{*}{$\mathrm{MC}$} \\
\hline & & & & \\
\hline \multicolumn{5}{|l|}{ Cenário mais provável } \\
\hline Receita de bonificação, $\mathrm{R} \$$ & 336,26 & 322,63 & 303,25 & 275,35 \\
\hline Custo dieta crescimento (fase I), R\$ & - & - & 42,24 & 45,57 \\
\hline Custo dieta crescimento (fase II), $\mathrm{R} \$$ & - & - & 35,63 & 38,81 \\
\hline Custo dieta terminação, $\mathrm{R} \$$ & 58,65 & 56,09 & 61,12 & 59,11 \\
\hline Custo total da dieta, $\mathrm{R} \$$ & 58,65 & 56,09 & 138,99 & 144,57 \\
\hline Custo da imunocastração, R\$ & 10,00 & - & 10,00 & - \\
\hline Custo total, $\mathrm{R} \$$ & 68,65 & 56,09 & 148,99 & 143,49 \\
\hline Lucro bruto, $\mathrm{R} \$$ & 267,61 & 266,54 & 154,26 & 131,87 \\
\hline Diferença monetária (IM-MC), R\$ & \multicolumn{2}{|c|}{1,07} & \multicolumn{2}{|c|}{22,39} \\
\hline Diferença relativa (IM-MC), \% & \multicolumn{2}{|c|}{0,04} & \multicolumn{2}{|c|}{16,98} \\
\hline \multicolumn{5}{|l|}{ Cenário otimista } \\
\hline Receita de bonificação, R\$ & 400,15 & 383,93 & 360,87 & 327,67 \\
\hline Custo dieta crescimento (fase I), R\$ & - & - & 38,02 & 41,01 \\
\hline Custo dieta crescimento (fase II), $\mathrm{R} \$$ & - & - & 32,07 & 34,93 \\
\hline Custo dieta terminação, $\mathrm{R} \$$ & 52,79 & 50,48 & 55,01 & 53,20 \\
\hline Custo total da dieta, $\mathrm{R} \$$ & 52,79 & 50,48 & 125,10 & 129,14 \\
\hline Custo da imunocastração, R\$ & 9,00 & - & 9,00 & - \\
\hline Custo total, R\$ & 61,79 & 50,48 & 134,10 & 129,14 \\
\hline Lucro bruto, $\mathrm{R} \$$ & 338,36 & 333,45 & 226,76 & 198,53 \\
\hline Diferença monetária (IM-MC), R\$ & \multicolumn{2}{|c|}{4,92} & \multicolumn{2}{|c|}{28,25} \\
\hline Diferença (IM-MC) \% & \multicolumn{2}{|c|}{1,47} & \multicolumn{2}{|c|}{14,23} \\
\hline \multicolumn{5}{|l|}{ Cenário pessimista } \\
\hline Receita de bonificação, R\$ & 272,37 & 261,33 & 245,64 & 223,03 \\
\hline Custo dieta crescimento (fase I), R\$ & - & - & 46,46 & 50,12 \\
\hline Custo dieta crescimento (fase II), R\$ & - & - & 39,20 & 42,69 \\
\hline Custo dieta terminação, $\mathrm{R} \$$ & 64,52 & 61,70 & 67,23 & 65,02 \\
\hline Custo total dieta, $\mathrm{R} \$$ & 64,52 & 61,70 & 152,89 & 157,83 \\
\hline Custo da imunocastração, R\$ & 11,00 & - & 11,00 & - \\
\hline Custo total, R\$ & 75,52 & 61,70 & 163,89 & 157,83 \\
\hline Lucro bruto, $\mathrm{R} \$$ & 196,85 & 199,63 & 81,74 & 65,20 \\
\hline Diferença monetária (IM-MC), R\$ & \multicolumn{2}{|c|}{$-2,78$} & \multicolumn{2}{|c|}{16,54} \\
\hline Diferença (IM-MC), \% & \multicolumn{2}{|c|}{$-1,39$} & \multicolumn{2}{|c|}{25,37} \\
\hline
\end{tabular}

IM (machos imunocastrados), MC (machos castrados cirurgicamente).

machos não castrados nas fases de crescimento I e II, em relação aos castrados cirurgicamente.

$\mathrm{Na}$ análise de sensibilidade para o cenário pessimista, no experimento I, o lucro bruto dos suínos IM foi similar ao dos suínos $\mathrm{MC}$, resultando numa diferença monetária de (-) R\$ 2,78 (-1,39\%), diferentemente do observado nos cenários mais provável e otimista. Isso se deve ao fato de que, no cenário pessimista, ocorreu diminuição na receita de bonificação, devido à redução no preço pago ao suíno vivo $\left(\mathrm{R} \$ \mathrm{~kg}^{-1}\right)$, diminuindo a diferença na receita entre os grupos IM e MC, associado ao aumento no custo da vacina.
No experimento II, a lucratividade dos animais IM em relação aos $\mathrm{MC}$ foi superior àquela observada no experimento I, em que o lucro bruto dos suínos IM apresentou superioridade de R \$ 16,54 $(25,37 \%)$ em relação aos MC. Esse resultado evidencia a importância de analisar todo o processo de produção, pois os ganhos obtidos nas fases de crescimento I e II influenciaram diretamente no lucro bruto. A maior lucratividade para os animais imunocastrados do experimento II, no cenário pessimista em relação aos demais cenários, pode ser explicada principalmente pelo aumento do custo da dieta, variável que representa a maior parte dos custos de produção. 
No presente estudo, os animais imunocastrados apresentaram significativamente melhor conversão alimentar na fase de crescimento I, menor consumo de ração na fase crescimento II, melhor conversão alimentar e maior ganho de peso diário na fase de terminação. A relação de troca $(\mathrm{kg}$ de suíno por kg de ração) foi menor, impactando diretamente na diminuição dos custos e aumentando a lucratividade.

Mesmo em condições de mercado desfavoráveis, a imunocastração foi mais lucrativa, diminuindo os custos com a alimentação, desde que as fases de crescimento I e II sejam incorporadas nos cálculos. As diferenças de custos entre os sistemas de produção dos animais IM e MC ocorreu, no presente estudo, sobre o consumo de ração e o custo da vacina. Como os animais do grupo IM permanecem não castrados durante as fases de crescimento I e II, apresentaram consumo superior aos MC, porém, esse custo é compensado financeiramente devido ao melhor desempenho. Por sua vez, o custo da vacina implica diretamente o custo de produção dos IM, em que a redução no custo da vacina aumenta o ganho por cabeça.

\section{CONCLUSÃO}

Suínos não castrados apresentam menor consumo de ração e melhor conversão alimentar nas fases de crescimento em relação aos castrados cirurgicamente. $\mathrm{Na}$ fase de terminação, suínos IM apresentam melhor ganho de peso, conversão alimentar, rendimento de carcaça e porcentagem de carne em relação aos MC. A utilização de machos IM aumenta a lucratividade na produção de suínos. A análise de lucratividade deve considerar todas as fases de criação, uma vez que o desempenho diferencial dos suínos não castrados na fase de crescimento influencia economicamente os resultados da produção.

\section{COMITÊ DE ÉTICA E BIOSSEGURANÇA}

Declaramos que os animais utilizados em experimentação que originou o artigo "Avaliação técnicoeconômica de suínos machos imuno e cirurgicamente castrados", submetido para publicação na revista Ciência Rural, foram criados sob os princípios do bem estar animal. $\mathrm{O}$ abate foi realizado em frigorífico comercial com inspeção federal respeitando a legislação quanto ao tempo de descanso, insensibilização e sangria.

\section{REFERÊNCIAS}

BONNEAU, M. Use of entire males for pig meat in the European Union. Meat Science, v.49, p.S257-S272, 1998. Disponível em: <http://www.sciencedirect.com/science/article/pii/
S0309174098900535>. Acesso em: 12 nov. 2011. doi: 10.1016/ S0309-1740(98)90053-5.

CAMPBELL, R.G. et al. Effects of sex and energy intake between 48 and $90 \mathrm{~kg}$ live weight on protein deposition in growing pigs. Animal Production, v.40, p.497-503, 1985. Disponível em: $<$ http://journals.cambridge.org/abstract_S0003356100040198>. Acesso em: 14 maio, 2013. doi: $\bar{h}$ ttp://dx.doi.org/10.1017/ S0003356100040198.

CEPEA. Boletim do suíno, n.16, p.2, 2011. Disponível em: <http:// www.cepea.esalq.usp.br/comunicacao/CepeaBoletimSuino14. pdf>. Acesso: 6 dez. 2011.

CLAUS, R. et al. Short-term endocrine and metabolic reactions before and after second immunization against GnRH in boars. Vaccine, v.25, p.4689-4696, 2007. Disponível em: <http://www. sciencedirect.com/science/article/pii/S0264410X07004045>. Acesso em: 16 maio, 2013. doi:10.1016/j.vaccine.2007.04.009.

GITMAN, L.J. Princípios da administração financeira essencial. 2.ed. Porto Alegre: Artmed $\AA$, 2000. 536p.

GUIDONI, A.L. Melhoria de processos para tipificação e valorização de carcaças suínas no Brasil. In: CONFERÊNCIA INTERNACIONAL VIRTUAL SOBRE QUALIDADE DE CARNE SUÍNA, 2000, Concórdia. Anais eletrônicos... Concórdia: CNPSA, 2000. p.221-234. Disponível em: <http:// www.cnpsa.embrapa.br/sgc/sgc_publicacoes/anais00cv_guidoni_ pt.pdf>. Acesso em: 6 nov. 2011.

JAROS, P. et al. Effect of active immunization against GnRH on androstenone concentration, growth performance and carcass quality in intact male pigs. Livestock Production Science, v.92, p.31-38, 2005. Disponível em: <http://www.sciencedirect.com/ science/article/pii/S0301622604001241>. Acesso em: 23 nov. 2011. doi: 10.1016/j.livprodsci.2004.07.011.

METZ, C.; CLAUS, R. Active immunization of boars against GnRH does not affect growth hormone but lowers IGF-I in plasma. Livestock Production Science, v.81, p.129-137, 2003. Disponível em: <http://www.sciencedirect.com/science/article/pii/ S0301622602003020>. Acesso em: 11 nov. 2011. doi: 10.1016/ S0301-6226(02)00302-0.

PATTERSON, R.L.S. 5 $\alpha$-Androst-16-en-3-one: compound responsible for taint in boar taint. Journal Science Food Agriculture, v.68, p.31, 1968. Disponível em: <http:// onlinelibrary.wiley.com/doi/10.1002/jsfa.2740190107/abstract?d eniedAccessCustomisedMessage $=\&$ userIsAuthenticated $=$ false $>$. Acesso em: 13 set. 2011. doi: 10.1002/jsfa.2740190107.

PAULY, C. et al. Growth performance, carcass characteristics and meat quality of group-penned surgically castrated, immunocastrated $\left(\operatorname{Improvac}^{\circledR}\right)$ and entire male pigs and individually penned entire male pigs. Animal Consortium, v.3, p.1057-1066, 2009. Disponível em: <http://journals.cambridge.org/download.php?fil $\mathrm{e}=\%$ 2FANM\%2FANM3_07\%2FS1751731109004418a.pdf\&cod $\mathrm{e}=63629576371 \mathrm{ddc} 0 \mathrm{~b} 81 \mathrm{bc} 6 \mathrm{fe} 8 \mathrm{a} 66 \mathrm{~d} 382 \mathrm{c}>$. Acesso em: $18 \mathrm{dez}$. 2011. doi: $10.1017 / \mathrm{S} 1751731109004418$.

RYDHMER, L. et al. Immunocastration reduces aggressive and sexual behaviour in male pigs. Animal Consortium, v.4, p.965-972, 2010. Disponível em: <http://journals.cambridge. org/download.php?file=\%2FANM\%2FANM4_06\%2FS1751731 
11000011Xa.pdf\&code $=\mathrm{d} 4243 \mathrm{f7} 7 \mathrm{ea} 140 \mathrm{~d} 967 \mathrm{fc} 0936 \mathrm{~b} 8 \mathrm{a} 4 \mathrm{~b} 398 \mathrm{c}>$. Acesso em: 26 set. 2011. doi: 10.1017/S175173111000011X.

XUE, J.L. et al. Performance, carcass, and meat quality advantages of boars over barrows: a literature review. Swine Health and Production, v.5, p.21-28, 1997. Disponível em: <https://www. aasv.org/shap/issues/v5n1/v5n1p21.pdf>. Acesso em: 14 nov. 2011.
ZAMARATSKAIA, G. et al. Effect of a Gonadotropin-releasing hormone vaccine (ImprovacTM) on steroid hormones, boar taint compounds and performance in entire male pigs. Reproduction in Domestic Animals, v.43, p.351-359, 2008. Disponível em: <http:// onlinelibrary.wiley.com/doi/10.1111/j.1439-0531.2007.00914.x/ abstract?deniedAccessCustomisedMessage $=$ \&userIs Authenti cated $=$ false $>$. Acesso em: 22 dez. 2011. doi: 10.1111/j.14390531.2007.00914.x. 